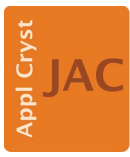

JOURNAL OF

APPLIED

CRYSTALLOGRAPHY

ISSN 1600-5767

Received 22 May 2017

Accepted 24 July 2017

Edited by G. Renaud, CEA-Grenoble DSM/ INAC/SP2M/NRS, Grenoble, France

Keywords: grain/twin boundary; transformation matrices; scanning transmission electron microscopy; GaAs; face-centred cubic zincblende nanomaterials.

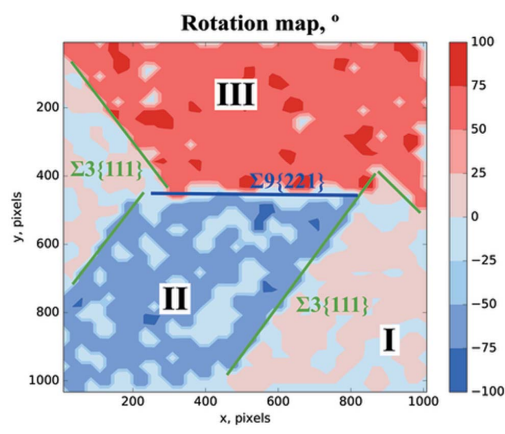

C 2017 International Union of Crystallography

\section{A tool for automatic recognition of [110] tilt grain boundaries in zincblende-type crystals}

\author{
Roksolana Kozak, ${ }^{\text {a* }}$ Fiodar Kurdzesau, ${ }^{\text {b }}$ Ivan Prieto, ${ }^{\mathrm{a}, \mathrm{c}}$ Oliver Skibitzki, ${ }^{\mathrm{d}}$ Thomas \\ Schroeder, ${ }^{\mathrm{d}, \mathrm{e}}$ Yadira Arroyo Rojas Dasilva, ${ }^{a}$ Rolf Erni, ${ }^{a}$ Hans von Känel ${ }^{\mathrm{a}, \mathrm{c}}$ and \\ Marta D. Rossell ${ }^{a}$
}

aElectron Microscopy Center, Empa, Swiss Federal Laboratories for Materials Science and Technology, Überlandstrasse 129, Dübendorf, CH-8600, Switzerland, 'bMAS 'Medical Physics', ETH Zürich, Gloriastrasse 35, Zürich, CH-8092, Switzerland, 'Laboratory for Solid State Physics, ETH Zürich, Otto-Stern-Weg 1, Zürich, CH-8093, Switzerland, dIHP, Im Technologiepark 25, Frankfurt (Oder), 15236, Germany, and $\mathbf{e}$ Institute of Physics, Brandenburgische Technische Universitaet, Konrad-Zuse Strasse 1, Cottbus, 03046, Germany. *Correspondence e-mail: roksolana.kozak@empa.ch

The local atomic structure of [110] tilt grain boundaries (GBs) formed in $\sim 100 \mathrm{~nm}$-sized GaAs nanocrystals, which crystallize in the non-centrosymmetric zincblende-type structure with face-centred cubic lattice symmetry, was imaged and analysed by means of high-resolution high-angle annular dark-field scanning transmission electron microscopy (HAADF-STEM). The nanocrystals were grown by metal-organic vapour phase epitaxy on top of (001) Si nanotips embedded in an oxide matrix. This paper introduces an automatic analysis method and corresponding processing tool for the identification of the GBs. The method comprises (i) extraction of crystallographic parameters, i.e. misorientation angles and transformation matrices for the different crystal parts (grains/ twins) observed by HAADF-STEM, and (ii) determination of their common plane(s) by modelling all possible intersections of the corresponding threedimensional reciprocal lattices. The structural unit model is also used to characterize the GB structures and to validate the data obtained by the developed algorithm.

\section{Introduction}

Nanoscale GaAs/Si heterostructures are expected to enable the development of new miniaturized devices combining the advantageous properties and functionalities of III-V compound semiconductors with the CMOS-compatible silicon technology (Volz et al., 2011). High-quality GaAs nanocrystals on top of nano-patterned $\mathrm{Si}(001)$ substrates may, for example, lead to high-speed field-effect transistors (Bartolomeo et al., 2017), solar cells (Fan et al., 1982) or lasers (Liang \& Bowers, 2010).

The epitaxial growth of GaAs with the zincblende/sphalerite $\mathrm{ZnS}$-type structure [space group $F \overline{4} 3 m$, Pearson symbol $c F 8$, lattice parameter $a=0.5654 \mathrm{~nm}$, with Ga at the Wyckoff position (4a) 0, 0, 0 and As at (4c) $\frac{1}{4}, \frac{1}{4}, \frac{1}{4}$ (Villars \& Cenzual, 2016/17)] on Si with the diamond C-type structure $[F d \overline{3} m, c F 8$, $a=0.5430 \mathrm{~nm}$, with two face-centred cubic (f.c.c.) sublattices shifted by $\frac{1}{4}, \frac{1}{4}, \frac{1}{4}$ along the body diagonal (Villars \& Cenzual, 2016/17)] is, however, challenging because of the significant lattice mismatch of $4.1 \%$ and the difference in thermal expansion coefficients (Bolkhovityanov \& Pchelyakov, 2008; Prieto, Kozak, Skibitzki, Rossell, Zaumseil et al., 2017; Skibitzki et al., 2017). This leads to the formation of various crystal imperfections (dislocations, stacking faults, twins etc.) (Kozak et al., 2017; Arroyo Rojas Dasilva et al., 2016; Stolz et al., 1988), which greatly influence the performance of the 
nano-devices in a detrimental way. In particular, grain boundaries (GBs) are defects acting as charge barriers and recombination centres in semiconductors (Dhanaraj et al., 2010). They can lead to low optical absorption, poor carrier mobility and reduced lifetime (i.e. high resistivity) of the optoelectronic materials. In addition, these defects serve as traps for impurities, which might degrade the optical and electrical properties of the devices (Visoly-Fisher et al., 2006).

However, the performance of devices can also be improved by controlling the type, orientation and frequency of occurrence as well as the distribution of the GBs present in the synthesized structures (Raghunathan et al., 2014). This kind of GB engineering (Watanabe, 1985, 2011) allows one to optimize the properties of known materials and to design new ones with better physical characteristics such as opto-electronic performance. It is therefore crucial to be able to identify and characterize the local GB structures at the atomic scale.

From a crystallographic point of view, a GB represents a two-dimensional defect (Holt \& Yacobi, 2007). It is usually formed during the crystal growth under insufficiently optimized conditions (temperature, presence of impurities, surface structure etc.). A GB is created by a change of the spatial orientation of the crystal lattice, leading to an interface between two differently oriented parts of the same crystal (Thibault et al., 2000). It can be fully described by three crystallographic parameters (Priester, 2013): the rotation angle $\theta$ between the two crystal parts forming the GB, the corresponding rotation axis $[u v w]$ defined by its direction cosines and the GB plane, defined by its normal vector $\mathbf{n}$

(a)
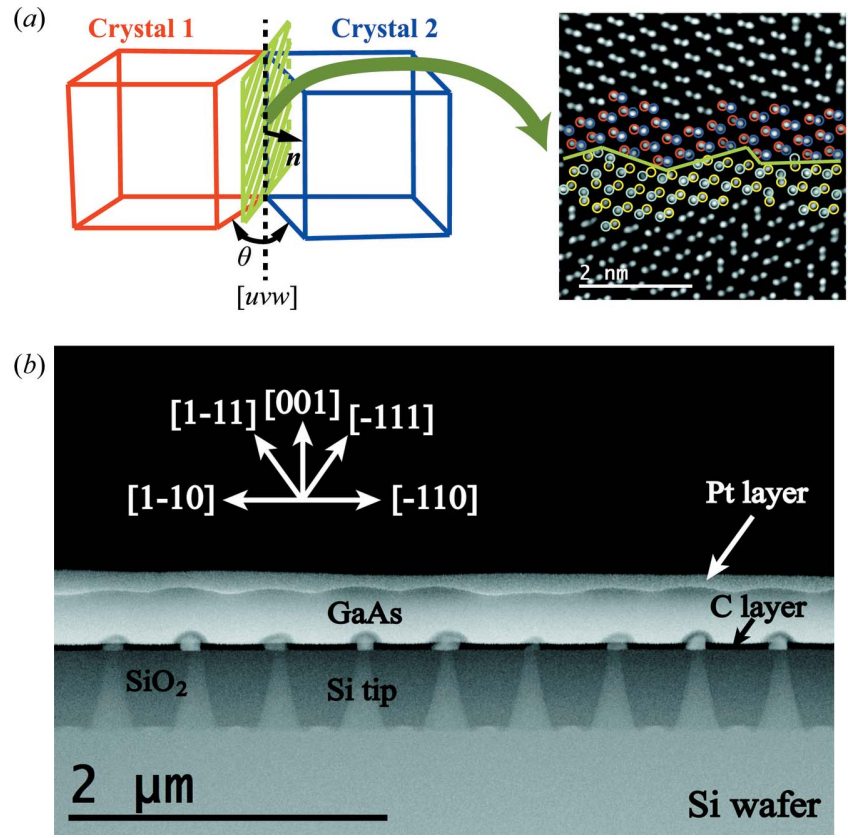

Figure 1

(a) Schematic image illustrating the formation of a GB at the intersection of two crystals, where $\theta$ describes the rotation angle, $[u v w]$ is the rotation axis and $\mathbf{n}$ is the normal vector to the GB plane. (b) Low-magnification HAADF-STEM image of the $\sim 100 \mathrm{~nm}$ GaAs nanocrystals grown on top of the (001) Si nanotips.
(Fig. 1a). They give five macroscopic degrees of freedom for this two-dimensional defect, which are enough to provide a complete thermodynamic modelling of the system state. The $\Sigma$ value of the $\mathrm{GB}$ reflects the volume fraction of the coincident site lattice (CSL) (Cayron, 2007; Grimmer et al., 1974). Following the common nomenclature, a GB is called a 'tilt GB' if its rotation axis lies in the boundary plane and a 'twist GB' if this axis is perpendicular to the boundary plane. Any other position can be referred to as a mixed tilt/twist GB (Priester, 2013). If the angle between the lattice vectors and the boundary on both sides of a tilt GB is the same, and consequently the GB has the same Miller indices in both crystal parts (i.e. $\{h k l\}_{\mathrm{I}}=\{h k l\}_{\mathrm{II}}$ ), the GB is called 'symmetric'. Conversely, when the corresponding angles are different and $\{h k l\}_{\mathrm{I}} \neq\{h k l\}_{\mathrm{II}}$, the GB is referred to as 'asymmetric' (Priester, 2013; Nebel \& Ristein, 2003). By definition, the first-order twin boundary $\Sigma 3$ is produced by a crystal rotation around one [111] axis. Repeated rotation(s) around this [111] axis or around a different [111] axis (axes) results in second (higher)order twins (Holt \& Yacobi, 2007).

Recently, atomic structure analysis of GBs has become possible due to a huge breakthrough in scanning transmission electron microscopy (STEM). The development of correctors for lens aberrations has enabled higher spatial resolution (below $0.08 \mathrm{~nm}$ ) with increased image contrast, allowing for the direct visualization of crystal defects (Erni et al., 2009; Pennycook \& Nellist, 2011). Here, we present a study of [110] tilt GBs formed in GaAs nanocrystals of $\sim 100 \mathrm{~nm}$ in size. The nanocrystals were grown on $\mathrm{Si}(001)$ nanotips embedded in an oxide matrix (Fig. $1 b$ ) by metal-organic vapour phase epitaxy (MOVPE) and imaged by high-resolution high-angle annular dark-field (HAADF)-STEM. We discuss an automated analysis method and corresponding image processing tool developed for the determination of the GB plane at the atomic scale.

\section{Experimental}

The initial nanotip patterned (001) Si substrates were produced using a state-of-the-art $0.13 \mu \mathrm{m}$ BiCMOS pilot line technology platform for $200 \mathrm{~mm}$ silicon wafers. The main steps for the fabrication of the substrates include deposition of $\mathrm{SiO}_{2}$ and $\mathrm{Si}_{3} \mathrm{~N}_{4}$, lithography, dry and wet etching, chemical mechanical planarization, and final wet cleaning. More details are given by Skibitzki et al. (2017) and Niu et al. (2016).

An epitaxial growth of $\mathrm{GaAs}$ nanocrystals on top of $\mathrm{Si}$ nanotips was performed. Firstly, cleaning in isopropanol and in Piranha was done, and the last step prior to the growth consisted of a $0.6 \%$ HF dip to remove the native oxide on the tips. The growth was carried out at $843 \mathrm{~K}$ in a MOVPE AIXTRON 200/4 reactor using arsine $\left(\mathrm{AsH}_{3}\right)$ and TMGa [trimethylgallium, $\mathrm{Ga}\left(\mathrm{CH}_{3}\right)_{3}$ ] as $\mathrm{As}$ and $\mathrm{Ga}$ precursors, respectively. Prior to GaAs deposition, an in situ As passivation at $1053 \mathrm{~K}$ was performed. More specific MOVPE growth parameters can be found elsewhere (Prieto, Kozak, Skibitzki, Rossell, Schroeder et al., 2017; Prieto, Kozak, Skibitzki, Rossell, Zaumseil et al., 2017). 
For the electron microscopy studies of the GaAs/Si nanostructures, several lamellae (see Fig. $1 b$ ) were prepared by means of an FEI Helios Nanolab 450S focused ion beam instrument operated at ion-beam accelerating voltages of 5$30 \mathrm{kV}$. Atomic resolution imaging for structural characterization was performed by STEM. These measurements were carried out using a double spherical aberration corrected JEOL JEM-ARM200F microscope operated at $200 \mathrm{kV}$. Images were acquired in STEM mode with a probe semiconvergence angle set to $25.3 \mathrm{mrad}$, which yields a probe size of about $80 \mathrm{pm}$. The annular semidetection range of the annular dark-field detector was set to collect electrons scattered between 90 and $370 \mathrm{mrad}$.

\section{Results and discussion}

Many different atomic structures for the $\Sigma 9 \mathrm{~GB}$ in diamond/ zincblende films have been reported so far (Nebel \& Ristein, 2003). A typical example of a boundary observed in a $\sim 100 \mathrm{~nm}$ GaAs nanocrystal grown on top of a (001) Si nanotip is illustrated in the high-resolution HAADF-STEM image in Fig. 2(a). Three theoretical concepts, which are complementary and very much overlap with each other, can be considered in order to describe its structure (Thibault et al., 2000). They represent geometrical, dislocation and structural unit (SU) models. In the following, the GB structures will be identified and characterized using our own automated analysis approach [the corresponding code has been written in the Python 2.7 programming language using the standard NumPy (http://www.numpy.org/) and SciPy (https://www.scipy.org/) libraries and graphical support from the Matplotlib (https:// matplotlib.org/) module, and is available on request by contacting the authors] discussed below and the standard SU model (see Fig. 2b), which is given for comparison (Thibault et al., 1993, 2000).

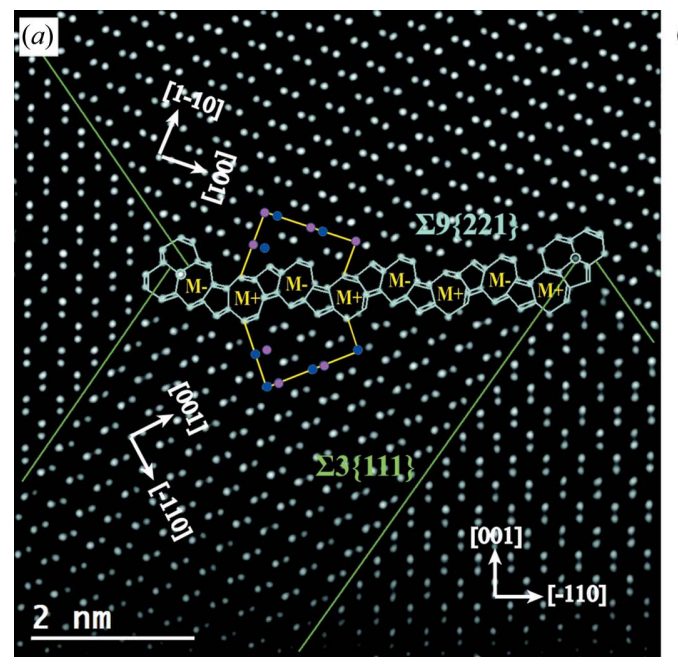

(b)<smiles>Cc1ccccc1CC1CCCCC1</smiles>
C

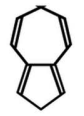

$\mathbf{L}$
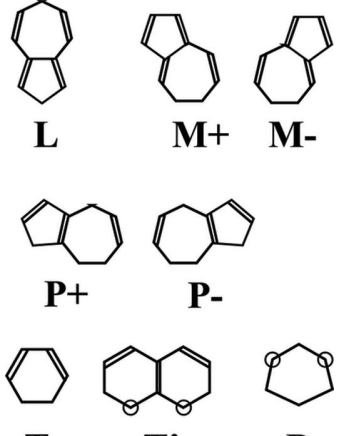

$\mathbf{T}$

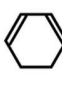

$\mathbf{T}^{\prime}$
In order to determine the main crystallographic parameters (i.e. rotation angle about [110] and shared plane) describing the GB structure in the GaAs nanocrystals and to derive an atomic model of the GB, we focus on the identification of transformation matrices from the high-resolution HAADFSTEM image for the different crystal parts (grains/twins). An application of matrices for the analysis of rotational twins has been reported previously (Calbick \& Marcus, 1967; Li \& Yan, 1983; Bleris \& Karakostas, 1989). The transformation matrix reflects the crystal's symmetry operation(s) and links one set of coordinates (e.g. main growth direction noted as reference) with another one (the twinned coordinate system) (De Graef \& McHenry, 2012; Cayron, 2007; Hovmöller, 1981).

Prior to data processing a Gaussian low-pass filter for noise reduction was applied to the image using the DigitalMicrograph software package (http://www.gatan.com/products/temanalysis/gatan-microscopy-suite-software). As a first step of the analysis, peak positions in the high-resolution HAADFSTEM image, shown as red dots in Figs. 3(a) and 3(b), are identified by a local maximum search method using an intensity cutoff value defined as the sum of a selected threshold range around the intensity mean value of the image. Then, each peak is precisely fitted with a two-dimensional Gaussian algorithm, similarly to the approach reported by Nord et al. (2017), mostly considering a $17 \times 17$ pixel block (which can be adjusted depending on the image scale) around the found local maximum position. Next, the pairs of closest peaks (i.e. dumbbell atomic columns) are identified by a minimum distance analysis. Each atomic pair is considered as one single vector directed from the lighter (Ga) atomic column to the heavier (As) one, having a specific orientation with respect to the vertical axis of the HAADF-STEM image. Thus, the orientation of these individual vectors defines the orientation of the different crystal parts to which they belong (see Fig. $3 b$ ). This approach is similar to the polarity determination within nanowire structures (Hertog et al., 2011; De la Mata et al., 2012). The misorientation angle $\theta$, i.e. the acute angle between the different crystal parts rotated about the [110] axis, can be found as the difference between the preferred orientations given by the vectors within the twin and those of the reference part of the crystal, i.e. grown epitaxially onto the (001) plane of the $\mathrm{Si}$ substrate. The $\theta$ calculation algorithm includes the representation of all dumbbell orientation angles of the HAADFSTEM image in one histogram with the subsequent identification of the local maxima, i.e. the preferred orientation angles for the reference and the twin crystals, applying a one-dimensional Gaussian fit and taking their difference (Fig. 3c). Lastly, the extracted geometrical parameters for each twin (crystallographic orientation and transformation matrix) are applied to model the three-dimensional reciprocal patterns (Fig. $3 d$ ) in order to find their possible common planes. Thus, the $\left(h_{1} k_{1} l_{1}\right)$ and

Figure 2

(a) High-resolution HAADF-STEM image presenting symmetric $\Sigma 9\{221\}$ (marked blue) and $\Sigma 3\{111\}$ (green lines) GBs. The GaAs unit cells at each side of the boundary are displayed as ball-and-stick models with the Ga and As columns shown in magenta and blue, respectively. (b) Models of known SUs used for the description of the [110] tilt GBs in zincblende-type semiconductors (Thibault et al., 1993, 2000). 
$\left(h_{2} k_{2} l_{2}\right)$ planes can be described as the intersection of the two corresponding lattices in reciprocal space:

$$
\left[R\left(\theta_{1}\right)\right]\left[A_{0}\right]\left(\begin{array}{l}
h_{1} \\
k_{1} \\
l_{1}
\end{array}\right)=\left[R\left(\theta_{2}\right)\right]\left[A_{0}\right]\left(\begin{array}{c}
h_{2} \\
k_{2} \\
l_{2}
\end{array}\right),
$$

where $\left[A_{0}\right]$ is an initial reciprocal-space matrix for cubic crystals defined by

$$
\left[A_{0}\right]=\left(\begin{array}{ccc}
a & 0 & 0 \\
0 & a & 0 \\
0 & 0 & a
\end{array}\right)^{-1}=\left(\begin{array}{ccc}
a^{*} & 0 & 0 \\
0 & a^{*} & 0 \\
0 & 0 & a^{*}
\end{array}\right) .
$$

$\left[R\left(\theta_{1}\right)\right]$ and $\left[R\left(\theta_{2}\right)\right]$ are the rotation matrices along the [110] axis with known twin orientation angles $\theta_{1}$ and $\theta_{2}$, respectively (Kabsch, 1977, 1988; Higashi, 1990). Combining the found common planes [see the coloured lines in Figs. 4(a)-4(c)] with the analysed STEM image (Fig. $3 a$ ) one can easily reconstruct the right GB. The extracted angles have also been used to model each twin and the intersection boundaries in real space.
Simulated patterns for a coherent $\Sigma 3\{111\}$ - and an incoherent $\Sigma 9\{221\}$-type twin boundary are presented in Figs. $4(d)$ and 4(e), respectively. The scripts (written in Python 2.7) are available on request by contacting the authors.

The obtained results for the symmetric $\Sigma 9\{221\}$ GB, which was used as an example for demonstrating our algorithm (see Figs. 2-4), are summarized in Fig. 5. This second-order twin boundary is created as a result of the interaction of two coherent twins $\Sigma 3\{111\}$ at a triple junction under a $\sim 70^{\circ}$ angle (Nebel \& Ristein, 2003; Cho \& Carter, 2001). Following the standard SU analysis (see Fig. 5a), one can see that it is a highcoincidence [110] tilt GB consisting of five- and seven-atom rings building a zigzag chain, which is achieved by shifting the GB plane by one atomic column (see Fig. $5 a$ ). This boundary is constructed with only one type of SU $\{\mathrm{M}-\mathrm{M}+\}(-$ and + refer to a mirror symmetry with respect to the GB) periodically arranged (Thibault et al., 2000). Thus, the $\Sigma 9$ GB forms an angle $\theta$ of $\sim 39^{\circ}$ with the $\{110\}$ planes, suggesting the $\{221\}$ plane as the common plane, which is of a glide mirror type with a period $a / 2[1 \overline{1} 4]$. According to calculations performed
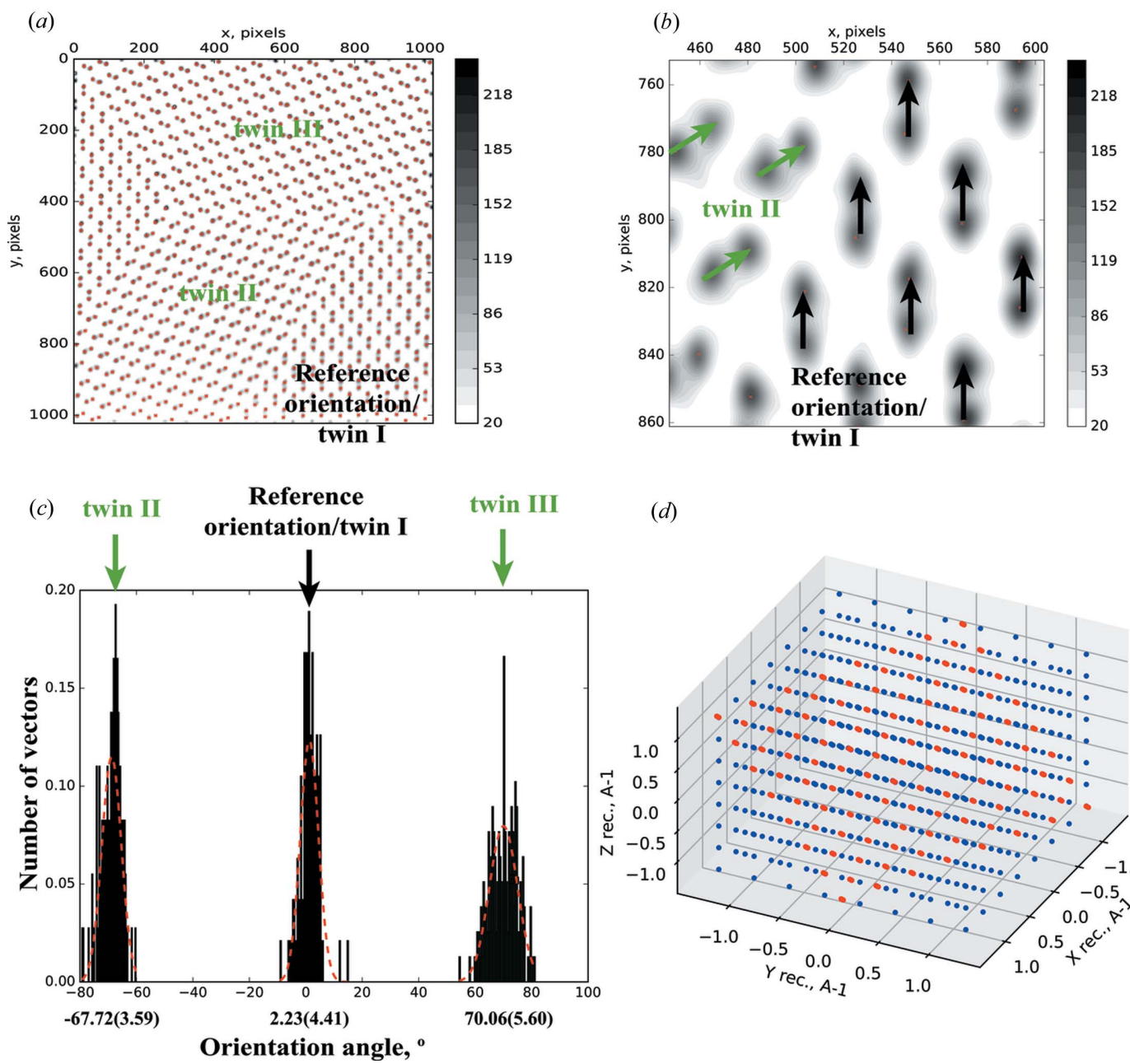

(d)

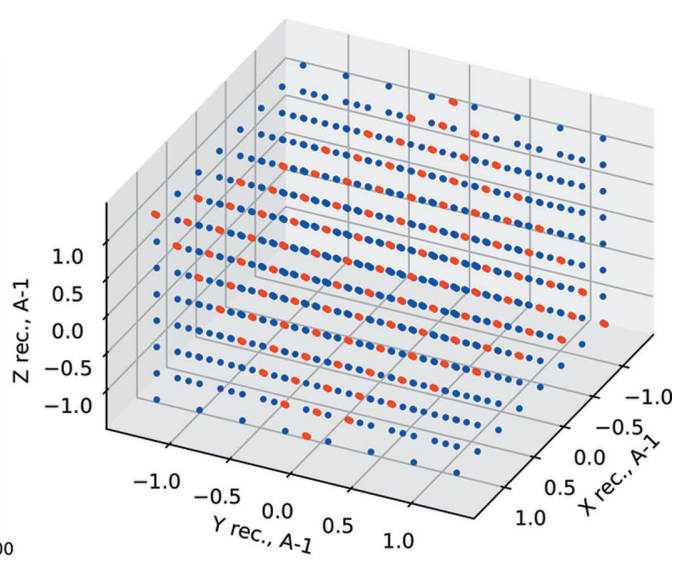

Figure 3

Orientation angle, ${ }^{\circ}$

(a) Automatic detection of the intensity peaks (marked with red dots) present in the HAADF-STEM image of Fig. 2(a). (b) Identification of the closest pairs of peaks as a single vector directed from the lighter $(\mathrm{Ga})$ to the heavier (As) atomic columns. Greyscale bars on the right indicate the pixel intensity. (c) Histogram presenting the extracted misorientation angles (in degrees) from the HAADF-STEM image in Fig. 2(a) for the different crystal parts. The dashed red line represents the result of the Gaussian fit. (d) Intersection of the three-dimensional reciprocal lattices for the reference crystal (twin I) in blue and twin II in red. 
for Si crystals it is a low-energy GB (Shenderova et al., 1999; Ratanaphan et al., 2014). The corresponding two-dimensional rotation map obtained as the final result of processing the image in Fig. 5(a) using the described algorithm is presented in Fig. 5(b). It shows the position of the three crystal parts (marked as I, II and III) clearly visualized as a function of the orientation angle between the dumbbells and the vertical [001] axis (see Fig. $3 c$ ). Thus, we obtain $\sim 0^{\circ}$ for the reference twin marked as I, around $-70^{\circ}$ for twin II and $\sim 70^{\circ}$ for twin III, which is in very good agreement with the HAADF-STEM (a)

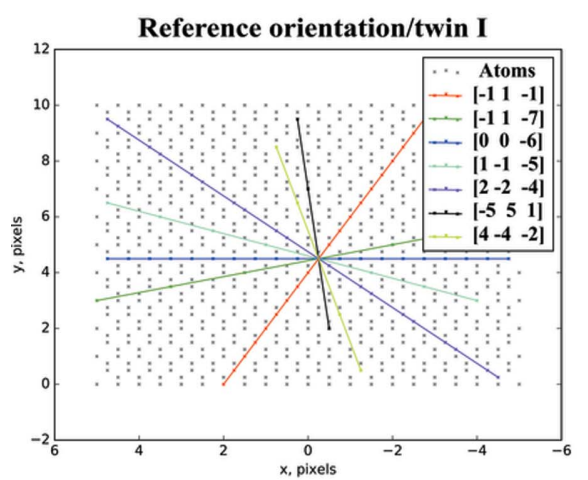

(d) (b)

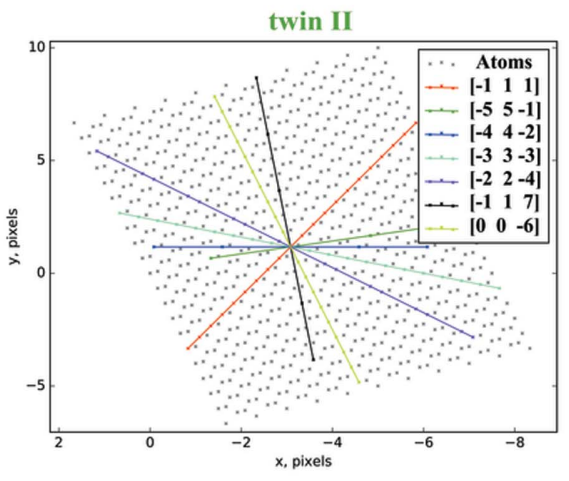

(c)

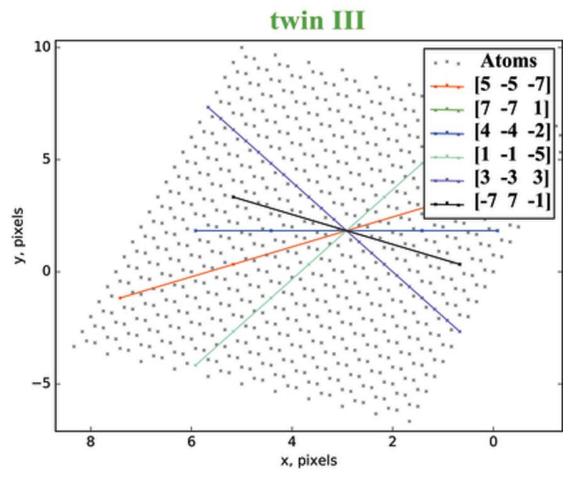

(e)
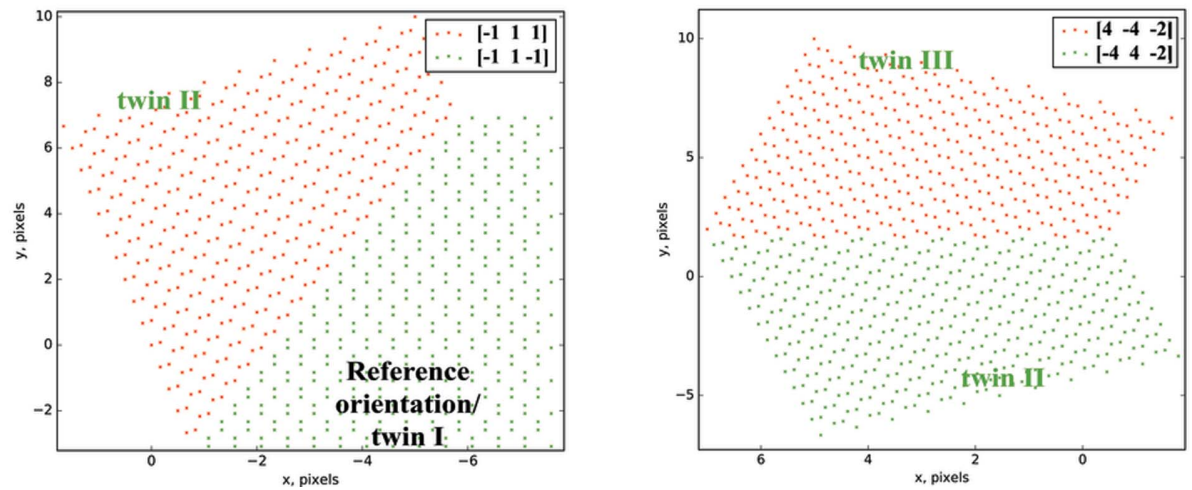

Figure 4

$(a),(b),(c)$ Simulated models of the reference crystal (twin I) and the other two twins (II and III), with coloured lines indicating the potential intersecting lattice planes. $(d),(e)$ Simulation of the coherent $\Sigma 3\{111\}$ (i.e. intersecting [111]][111] planes in II/I twin crystals) and incoherent $\Sigma 9\{221\}$ (i.e. intersecting [ $[\overline{4} 42] /[4 \overline{4} 2]$ planes in II/III twin crystals) twin boundaries.
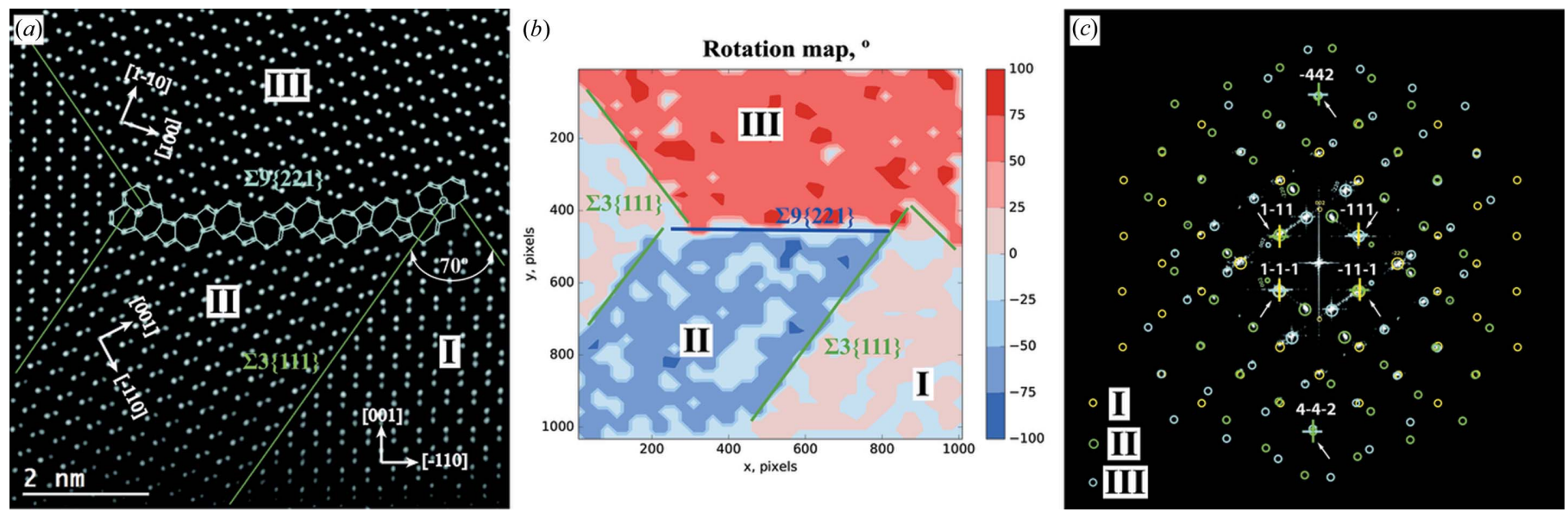

Figure 5

(a) High-resolution HAADF-STEM image presenting symmetric $\Sigma 9\{221\}$ (marked blue) and $\Sigma 3\{111\}$ (green lines) GBs for twins I (reference), II and III with overlaid SU analysis. (b) Corresponding lattice rotation map (in degrees) obtained by the automatic processing algorithm with the twins coloured according to their orientation. (c) Fourier transform of the image in panel ( $a$ ) with overlaid electron diffraction pattern simulated by the $J E M S$ software for the different crystal parts/grains (empty circles highlighted in different colours). The position of the common planes is shown with white arrows. 
image (Fig. 5a). Its Fourier transformation calculated with the image processing package DigitalMicrograph (Fig. 5c) was also compared with the electron diffraction pattern simulated by the JEMS software (Stadelmann, 2007) for each twin observed in Fig. 5(a) as a rotation by its orientation angle [see the coloured circles overlaid in Fig. 5(c)]. This can be assumed to be a simplified version of the three-dimensional representation depicted in Fig. 3(d). The intersections of the $(\overline{4} 4 \overline{2})_{\mathrm{II}}$, $(4 \overline{4} \overline{2})_{\mathrm{III}}$, and $(\overline{1} 1 \overline{1})_{\mathrm{I}},(\overline{1} 11)_{\mathrm{II}}$, and $(1 \overline{1} \overline{1})_{\mathrm{I}},(1 \overline{1} 1)_{\mathrm{III}}$ common planes observed from the modelling in Figs. $4(d)$ and $4(e)$ are then confirmed by visual overlapping of the corresponding diffraction peaks (see Fig. $5 c$ ).

Fig. 6(a) shows another case of a $\Sigma 9\{221\} \mathrm{GB}$, which is more complicated owing to the presence of stacking faults in twin II. According to the SU analysis, it results from the interaction of two $\Sigma 3\{111\}$ GBs under a $\sim 109^{\circ}$ angle (Nebel \& Ristein, 2003), and it is then built of five-, six- and seven-atom rings. The additional $T$ (six-atom ring) SU is associated with a sessile GB dislocation with Burgers vector $\mathbf{b}_{\mathrm{c}}=1 / 9 a[-221]$ (Thibault et al., 1993). The perfect $\mathbf{b}_{\mathrm{c}}$, which climbs along the GB, is formed by the dissociation of a $90^{\circ}$ Shockley partial dislocation when it reaches the GB (Bourret \& Bacmann, 1987; Elkajbaji \& Thibault-Desseaux, 1988) according to the following equation: $1 / 6 a[\overline{1} 12]=1 / 9 a[\overline{2} 21]+1 / 18 a[1 \overline{1} 4]$. At the places where the GB meets an intrinsic stacking fault the 'imperfect' $\mathbf{b}_{\mathrm{c}}$ is observed (Thibault-Desseaux et al., 1989). It is characterized by an $a / 2$ step (King \& Smith, 1980). The application of the developed processing algorithm to the image in Fig. 6(a) is shown as a final two-dimensional rotation map in Fig. 6(b), while the corresponding electron diffraction
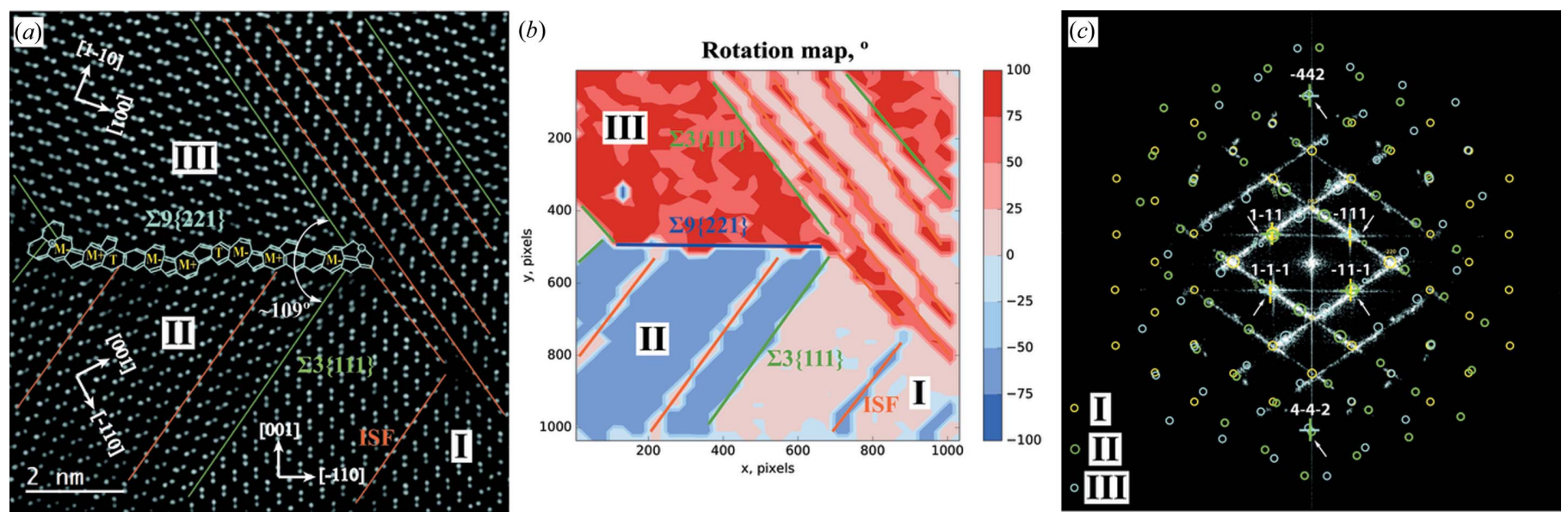

Figure 6

(a) High-resolution HAADF-STEM image presenting the interaction of two intrinsic stacking faults (red lines) with a $\Sigma 9\{221\}$ GB (in blue) between twins II and III with overlaid SU analysis. Green lines mark the $\Sigma 3\{111\}$ GBs. (b) Corresponding lattice rotation map (in degrees) obtained by the automatic processing algorithm. ISFs and ESFs are highlighted in red, and the different twins are coloured according to their orientation. (c) Fourier transform of the image in panel $(a)$ with overlaid electron diffraction pattern simulated by the JEMS software for the different crystal parts/grains (empty circles highlighted in different colours). The position of the common planes is shown with white arrows.
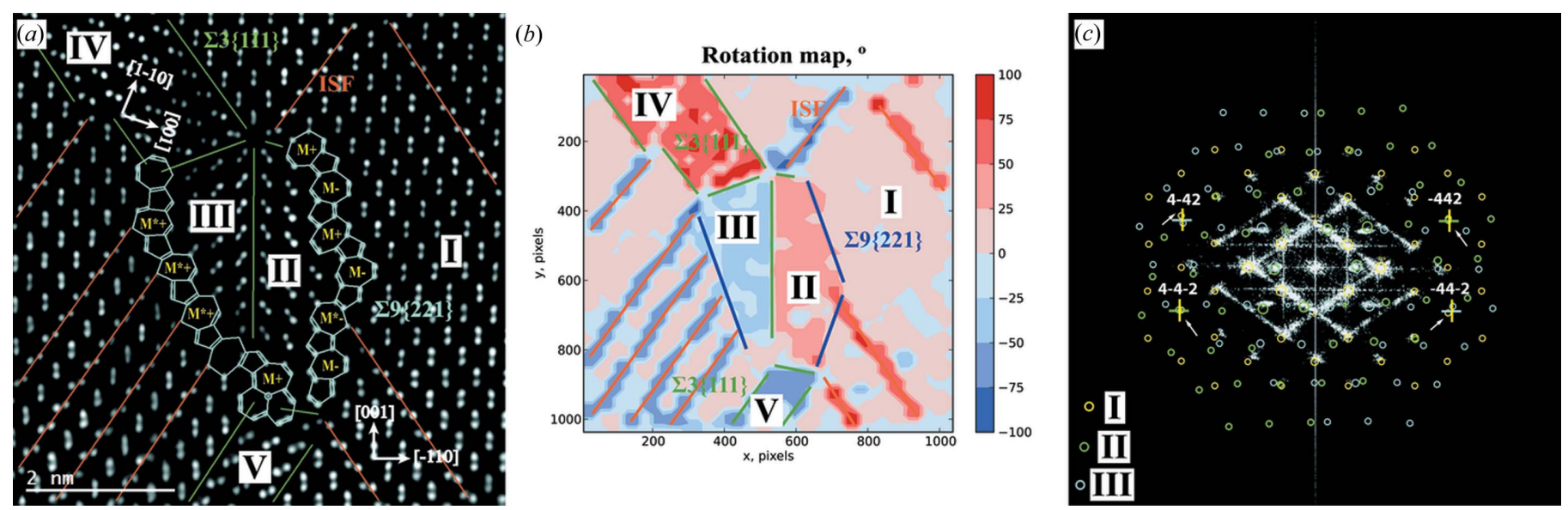

Figure 7

(a) High-resolution HAADF-STEM image presenting a fivefold twinning system including complex $\Sigma 9\{221\}$ (in blue) and $\Sigma 3\{111\}$ (in green) GBs with overlaid SU analysis. The red lines indicate the position of the ISFs. $(b)$ Corresponding lattice rotation map (in degrees) obtained by the automatic processing algorithm with coloured twins and GBs. $(c)$ Fourier transform of the image in panel $(a)$ with overlaid electron diffraction pattern simulated by the JEMS software for the different crystal parts/grains (empty circles highlighted in different colours). The position of the common planes is shown with white arrows. 

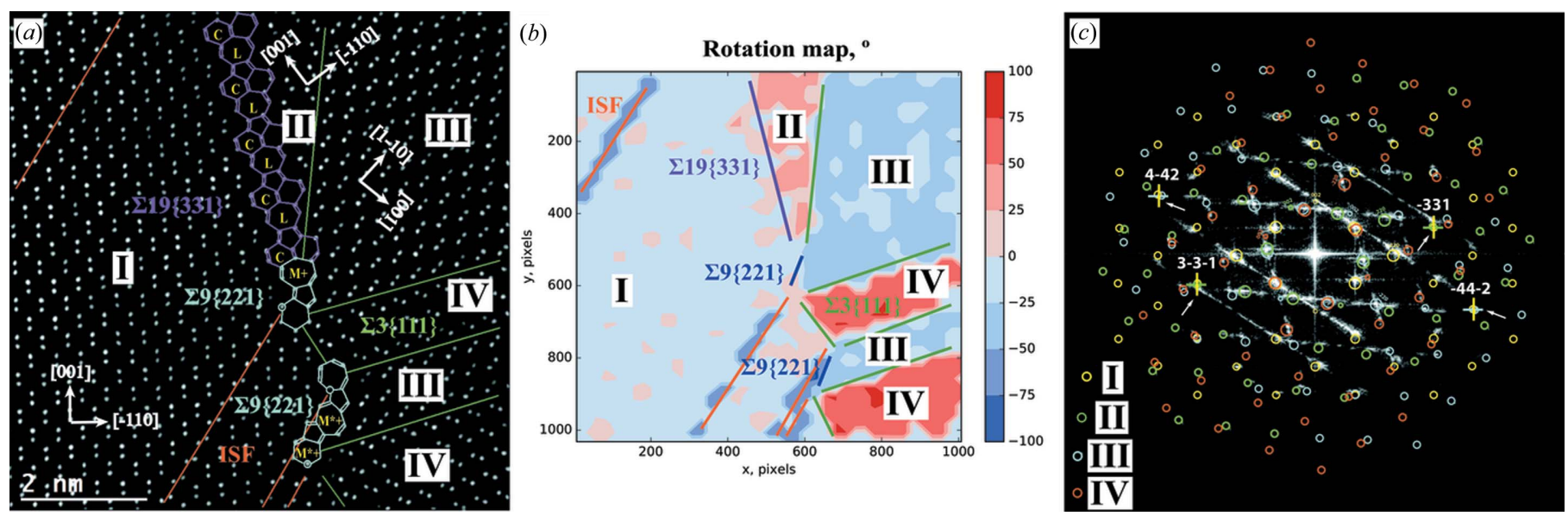

Figure 8

(a) High-resolution HAADF-STEM image presenting a fourfold twinning system with a symmetric $\Sigma 19\{331\}$ GB (in violet with overlaid SU analysis. Green and red lines mark the $\Sigma 3\{111\}$ GBs and ISFs, respectively. (b) Corresponding lattice rotation map (in degrees) obtained by the automatic processing algorithm with coloured twins and GBs. $(c)$ Fourier transform of the image in panel $(a)$ with overlaid electron diffraction pattern simulated by the JEMS software for the different crystal parts/grains (empty circles highlighted in different colours). The position of the common planes is shown with white arrows.

pattern simulated with the JEMS software (Stadelmann, 2007) for each observed twin (I, II and III) is displayed with coloured circles in Fig. 6(c). As one can see, the generated rotation map clearly indicates the observed twins, colouring them according to their orientation angle and visualizing the corresponding GB (see Fig. $6 b$ ). The found common planes $(\overline{4} 4 \overline{2})_{\mathrm{II}},(4 \overline{4} \overline{2})_{\mathrm{III}}$, and $(\overline{1} 1 \overline{1})_{\mathrm{I}},(\overline{1} 11)_{\mathrm{II}}$, and $(1 \overline{1} \overline{1})_{\mathrm{I}},(1 \overline{1} 1)_{\mathrm{III}}$ are also confirmed [their intersections are marked with crosses in Fig. 6(c)]. It should be emphasized that the developed algorithm is also suitable for the visualization of both intrinsic and extrinsic stacking faults (ISFs and ESFs, respectively) within the crystal (see Fig. 6b).

The developed approach can be efficiently used in the much more complex case of a $\Sigma 9\{221\}$ GB with five separated twins presented in Fig. 7(a). It is a zigzag boundary, which changes to a straight one at the places of interaction with an intrinsic stacking fault(s). They cause the rearrangement of a double bond in a seven-atom ring, leading to a slightly different SU (marked with an asterisk). The presence of extra $\mathrm{M}+$ or removal of $\mathrm{M}$ - SUs and vice versa introduces a glissile dislocation with $\mathbf{b}_{\mathrm{g}}=1 / 18 a[1 \overline{1} 4]$ as previously reported in the literature (Thibault et al., 1993). The corresponding generated rotation map (see Fig. $7 b$ ) efficiently reveals the areas of all five twins, colouring them according to their orientation angles with respect to the vertical [001] axis (they are around $0^{\circ}, \pm 39^{\circ}$ and $\pm 70^{\circ}$, respectively). Additionally, the different planar defects are indicated: the stacking faults (red lines), the $\Sigma 9\{221\}$ GBs (blue lines) between twins II, III and I, and the $\Sigma 3\{111\}$ GBs (green lines) for the IV/III, III/II, IV/I, V/I, II/I and II/V intersections. The identified GBs are also confirmed by the electron diffraction pattern simulated with the JEMS software (Stadelmann, 2007) for each twin observed in Fig. 7(a).

Besides the $\Sigma 9 \mathrm{~GB}$, a small-angle $\left(\theta \simeq 26.5^{\circ}\right)$ grain boundary $\Sigma 19\{331\}$ has been observed in the studied GaAs nanocrystals (Fig. 8a) and successfully processed with our algorithm (see Fig. 8b). Its structural model was predicted by Hornstra (1959). This symmetric GB is constructed of periodically arranged five-, seven- and a pair of six-atom rings, which correspond to $\mathrm{SU}\{\mathrm{L}\}$ and $\{\mathrm{C}\}$, respectively, i.e. an intermediate GB between two limiting $\Sigma 9$ and $\Sigma 1$ GBs. The unit $\{\mathrm{L}\}$ is the core of a Lomer edge dislocation with $\mathbf{b}=1 /$ 2a[110] (Thibault et al., 1993, 2000; Arroyo Rojas Dasilva et al., 2016). The $\Sigma 19\{331\}$ GB plane is of a mirror type with a period $a / 2[61 \overline{1}]$. The developed algorithm is capable of determining all four types of twins following their orientation angles and of visualizing the corresponding intersections also including the $(\overline{3} 31)_{\mathrm{I}}$ and $(\overline{3} 3 \overline{1})_{\mathrm{II}}$ common planes for the I/II boundary, marked with a violet line in Fig. 8(b). These GBs are also confirmed by the electron diffraction pattern simulated with the JEMS software (see Fig. 8c).

\section{Conclusions}

By means of high-resolution HAADF-STEM images, the local atomic arrangements in symmetric $\Sigma 9\{221\}$ and $\Sigma 19\{331\}$ [110] tilt grain boundaries present in $\sim 100 \mathrm{~nm}$ GaAs nanocrystals grown by MOVPE on top of (001) Si nanotips have been visualized and described. In order to determine the rotation angle(s) and GB plane(s) within the acquired STEM images, our own automatic analysis approach and a corresponding processing tool written in the Python 2.7 programming language have been developed. This approach has been subsequently applied and tested with several GBs. Its main processing steps are the following: peak search and refinement, extraction of the misorientation angles and transformation matrices for each twin within the analysed image by considering the closest atomic (dumbbell) peaks as a single vector following the polarity given by the direction from the lighter (Ga) atomic column to the heavier (As) one, and finding the common plane(s) by three-dimensional modelling of all possible intersections of the corresponding reciprocal 
lattices. The developed script can be used to analyse [110] tilt GBs in any f.c.c.-based zincblende material with any degree of complexity as long as dumbbell atomic columns are clearly resolved in the HAADF-STEM image. The standard SU model has also been used to characterize the analysed GB structures in order to validate the results obtained by the developed processing algorithm. It is remarkable that the applied tool is also capable of polarity assignment and visualizing other kinds of crystal defects such as intrinsic and extrinsic stacking faults, and it will be used in the future for fully automated defect analysis of high-resolution STEM images.

\section{Acknowledgements}

The authors would like to thank Professor G.-L. Bona for valuable suggestions and comments on the topic. The authors also thank the FIRST Center for Micro- and Nanoscience for making available its infrastructure. Access to the transmission electron microscopy facilities at IBM Research-Zürich, Switzerland, under the IBM/Empa Master Joint Development Agreement, is gratefully acknowledged.

\section{Funding information}

The following funding is acknowledged: Schweizerischer Nationalfonds zur Förderung der Wissenschaftlichen Forschung (award No. DACH 200021L-153558); Deutsche Forschungsgemeinschaft (award No. SCHR 1123/10-1).

\section{References}

Arroyo Rojas Dasilva, Y., Kozak, R., Erni, R. \& Rossell, M. D. (2017). Ultramicroscopy, 176, 11-22.

Bartolomeo, A. D., Giubileo, F., Luongo, G., Iemmo, L., Martucciello, N., Niu, G., Fraschke, M., Skibitzki, O., Schroeder, T. \& Lupina, G. (2017). 2D Materials, 4, 015024.

Bleris, G. L. \& Karakostas, Th. (1989). Acta Cryst. A45, 297-303.

Bolkhovityanov, Y. B. \& Pchelyakov, O. P. (2008). Phys.-Usp. 51, 437456.

Bourret, A. \& Bacmann, J. J. (1987). Rev. Phys. Appl. (Paris), 22, $563-$ 568.

Calbick, C. J. \& Marcus, R. B. (1967). Acta Cryst. 23, 12-17.

Cayron, C. (2007). Acta Cryst. A63, 11-29.

Cho, N. \& Carter, C. B. (2001). Jpn. J. Appl. Phys. 40, 4458-4465.

De Graef, M. \& McHenry, M. (2012). Structure of Materials: an Introduction to Crystallography, Diffraction and Symmetry. Cambridge University Press.

De la Mata, M., Magen, C., Gazquez, J., Utama, M. I. B., Heiss, M., Lopatin, S., Furtmayr, F., Fernández-Rojas, C. J., Peng, B., Morante, J. R., Rurali, R., Eickhoff, M., Fontcuberta i Morral, A., Xiong, Q. \& Arbiol, J. (2012). Nano Lett. 12, 2579-2586.

Dhanaraj, G., Byrappa, K., Prasad, V. \& Dudley, M. (2010). Editors. Springer Handbook of Crystal Growth. Berlin, Heidelberg: Springer-Verlag.

Elkajbaji, M. \& Thibault-Desseaux, J. (1988). Philos. Mag. A, 58, 325345.

Erni, R., Rossell, M. D., Kisielowski, C. \& Dahmen, U. (2009). Phys. Rev. Lett. 102, 096101.

Fan, J. C. C., Tsaur, B.-Y. \& Palm, B. J. (1982). Proceedings of the 16th Photovoltaic Specialists Conference, pp. 692-701. New York: IEEE Press.

Grimmer, H., Bollmann, W. \& Warrington, D. H. (1974). Acta Cryst. A30, 197-207.
Hertog, M. D., Elouneg-Jamroz, M., Bellet-Amalric, E., Bounouar, S., Bougerol, C., André, R., Genuist, Y., Poizat, J. P., Kheng, K. \& Tatarenko, S. (2011). J. Phys. Conf. Ser. 326, 012044.

Higashi, T. (1990). J. Appl. Cryst. 23, 253-257.

Holt, D. B. \& Yacobi, B. G. (2007). Extended Defects in Semiconductors: Electronic Properties, Device Effects and Structures. Cambridge University Press.

Hornstra, J. (1959). Physica, 25, 409-422.

Hovmöller, S. (1981). Rotation Matrices and Translation Vectors in Crystallography. IUCr Pamphlet No. 9, edited by C. A. Taylor. University College Cardiff Press.

Kabsch, W. (1977). J. Appl. Cryst. 10, 426-429.

Kabsch, W. (1988). J. Appl. Cryst. 21, 67-72.

King, A. H. \& Smith, D. A. (1980). Acta Cryst. A36, 335-343.

Kozak, R., Prieto, I., Arroyo Rojas Dasilva, Y., Erni, R., Skibitzki, O., Capellini, G., Schroeder, T., von Känel, H. \& Rossell, M. D. (2017). Philos. Mag. http://dx.doi.org/10.1080/14786435.2017.1355117.

Li, C. \& Yan, M. (1983). Mater. Sci. Eng. 57, 143-147.

Liang, D. \& Bowers, J. E. (2010). Nat. Photon. 4, 511-517.

Nebel, C. E. \& Ristein, J. (2003). Thin-Film Diamond I, Series in Semiconductors and Semimetals, Vol. 76. San Diego: Elsevier Academic Press.

Niu, G., Capellini, G., Schubert, M. A., Niermann, T., Zaumseil, P., Katzer, J., Krause, H. M., Skibitzki, O., Lehmann, M., Xie, Y. H., von Känel, H. \& Schroeder, T. (2016). Sci. Rep. 6, 1-11.

Nord, M., Vullum, P. E., MacLaren, I., Tybell, T. \& Holmestad, R. (2017). Adv. Struct. Chem. Imag. 3, 9.

Pennycook, S. J. \& Nellist, P. D. (2011). Scanning Transmission Electron Microscopy. New York: Springer.

Priester, L. (2013). Grain Boundaries - From Theory to Engineering. Dordrecht: Springer.

Prieto, I., Kozak, R., Skibitzki, O., Rossell, M. D., Schroeder, T., Erni, R. \& von Känel, H. (2017). Small, 13, 1603122.

Prieto, I., Kozak, R., Skibitzki, O., Rossell, M. D., Zaumseil, P., Capellini, G., Gini, E., Kunze, K., Rojas Dasilva, Y. A., Erni, R., Schroeder, T. \& von Känel, H. (2017). Nanotechnology, 28, 135701135708.

Raghunathan, R., Johlin, E. \& Grossman, J. C. (2014). Nano Lett. 14, 4943-4950.

Ratanaphan, S., Yoon, Y. \& Rohrer, G. S. (2014). J. Mater. Sci. 49, 4938-4945.

Shenderova, O. A., Brenner, D. W. \& Yang, L. H. (1999). Phys. Rev. $B, 60,7043-7052$.

Skibitzki, O., Prieto, I., Kozak, R., Capellini, G., Zaumseil, P., Arroyo Rojas Dasilva, Y., Rossell, M. D., Erni, R., von Känel, H. \& Schroeder, T. (2017). Nanotechnology, 28, 135301-135310.

Stadelmann, P. (2007). JEMS: Java Electron Microscopy Software, http://cime.epfl.ch.

Stolz, W., Guimaraes, F. E. G. \& Ploog, K. (1988). J. Appl. Phys. 63, 492-499.

Thibault, J., Putaux, J. L., Jacques, A., George, A., Michaud, H. M. \& Baillin, X. (1993). Mater. Sci. Eng. A, 164, 93-100.

Thibault, J., Rouviere, J.-L. \& Bourret, A. (2000). Handbook of Semiconductor Technology: Electronic Structure and Properties of Semiconductor, Vol. 1, edited by K. A. Jackson \& W. Schröter, ch. 7. Weinheim: Wiley-VCH Verlag GmbH.

Thibault-Desseaux, J., Putaux, J. L., Bourret, A. \& Kirchner, H. O. K. (1989). J. Phys. Fr. 50, 2525-2540.

Villars, P. \& Cenzual, K. (2016/17). Pearson's Crystal Data: Crystal Structure Database for Inorganic Compounds. Materials Park: ASM International.

Visoly Fisher, I., Cohen, S. R., Gartsman, K., Ruzin, A. \& Cahen, D. (2006). Adv. Funct. Mater. 16, 649-660.

Volz, K., Beyer, A., Witte, W., Ohlmann, J., Németh, I., Kunert, B. \& Stolz, W. (2011). J. Cryst. Growth, 315, 37-47.

Watanabe, T. (1985). J. Phys. Colloq. 46, C4-555-C4-564.

Watanabe, T. (2011). J. Mater. Sci. 46, 4095-4115. 